\title{
The Maya Behind Moyamoya-The Two Extremes of the Disease : Correspondence
}

\author{
Anita Choudhary • Naveen Sankhyan • Pratibha Singhi
}

Received: 9 October 2012 / Accepted: 22 January 2013 / Published online: 18 June 2013

(C) Dr. K C Chaudhuri Foundation 2013

To the Editor: We read with great interest the article by Vijayakanthi $\mathrm{N}$ et al. [1]. The brief report describes two patients of pediatric moyamoya disease with extreme presentation. However, we would like to point out certain issues relevant to the disease in question.

These presentations cannot be termed extreme as the natural history of moyamoya is variable. Disease progression can be slow, with rare, intermittent events, or fulminant, with rapid neurologic decline. In pediatric population, there are multitude of symptoms associated with moyamoya disease, including transient ischemic attack, ischemic strokes, intracranial hemorrhages, seizures, headaches, choreiform movements, and cognitive deficits. Recently researchers have also reported asymptomatic cases of moyamoya and approximately half of them remained clinically asymptomatic over a mean follow up period of $5.4 \mathrm{y}$ [2]. Thus, it is not unusual to see these variable presentations of moyamoya. The variations result possibly from the rapidity and extent of stenosis of intracranial vessels and the patient's ability to develop effective collaterals [3].

Authors have described that both patients had unilateral finding on angiography. As per the guidelines on the diagnosis and treatment of spontaneous occlusion of circle of willis (Moyamoya disease) unilateral involvement qualifies a diagnosis of a probable case of moyamoya disease instead of definitive case of moyamoya disease [4].

The authors have not mentioned, how was the diagnosis of moyamoya disease concluded in the absence of any collaterals in case-2. As per the diagnostic criteria presence of collaterals on angiography is essential for qualifying a diagnosis of moyamoya disease [4]. Further, how do the authors explain the bilateral findings on clinical examination

\footnotetext{
A. Choudhary $\cdot$ N. Sankhyan $\cdot$ P. Singhi $(\bowtie)$

Department of Pediatrics, Post Graduate Institute of Medical

Education and Research, Chandigarh 160012, India

e-mail: doctorpratibhasinghi@gmail.com
}

and MRI brain, but narrowing of only left internal carotid artery with normal right internal carotid artery.

The authors have quoted the study by Mugikura et al. [5] for explaining severe presentation in early onset $(<4 \mathrm{y}$ of age) moyamoya disease, but in the current case (2nd case) there was no involvement of posterior cerebral artery, thus whether this hypothesis of advanced posterior cerebral artery stenosis leading to severe clinical presentation can be applied to the present case is not clear. Further the hypothesis proposed by Mugikara $\mathrm{S}$ et al. also needs confirmation by prospective studies.

Finally, it is not clear whether the authors had excluded secondary causes of angiographic moya moya i.e., moya moya syndrome and whether these cases were indeed moya moya disease (idiopathic).

At the end "The Maya behind Moyamoya" still remains elusive.

\section{References}

1. Vijayakanthi N, Natarajan T. The maya behind moyamoya-The two extremes of the disease. Indian J Pediatr. 2012; doi:10.1007/ s12098-012-0813-x

2. Lin N, Baird L, Koss M, et al. Discovery of asymptomatic moyamoya arteriopathy in pediatric syndromic populations: radiographic and clinical progression. Neurosurg Focus. 2011; 31: E6.

3. Maki Y, Enomoto T. Moyamoya disease. Childs Nerv Syst. 1988; 4: 204-12.

4. Fukui M. Guidelines for the diagnosis and treatment of spontaneous occlusion of the circle of Willis ('Moyamoya' disease). Clin Neurol Neurosurg. 1997; 99: S238-40.

5. Mugikura S, Higano S, Shirane R, Fujimura M, Shimanuki Y, Takahashi S. Posterior circulation and high prevalence of ischemic stroke among young pediatric patients with Moyamoya disease: evidence of angiography-based differences by age at diagnosis. Am J Neuroradiol. 2011; 32: 192-8. 